\title{
In Praise of the \\ Disassembler
}

THERE'S MUCH

TO BE LEARNED

FROM THE LOWER-

LEVEL DETALLS OF

HARDWARE

Dear KV,

I've read enough of your columns to see that one of your frequent themes is that source code is meant to be read by people, including one's future selves, and that how that code is processed by an interpreter such as Python or a compiler is less important than making the code clear to the next reader. You seem to be saying that our tools will work out what we mean and that we should treat the interpreter or compiler as a black box that magically turns our source into running code. I feel you're ignoring an important part of understanding software, which is what happens when your compiled code executes on a machineafter all, no computer executes C, C++, or Rust directly; they're running a compiled binary. What happens when you have a bug that appears in the binary only because of a mistake by the compiler, linker, assembler, or other part of the tool chain, which must occur from time to time. What then?

Dissembling at the Back End

Dear Disassembling,

Indeed, there have been many people and many

movements within the software industry over the past 50 years that have shifted developers and development further away from machine code and assembly languageand not without good reasons. The abstractions of 
higher-level languages over the admittedly brief history of computing have allowed the explosion of software and services that nonprogrammers take for granted every day. Those of us who often work down in the bowels of technology know that these abstractions, and the movement of developers away from the machine, come with costs.

There are many problems in software systems that cannot be properly understood without a good understanding of the lower-level-some might argue the lowest-level-details of the machines we work on, and it shocks and angers me when I try to explain such things and get blank stares from people who have been in the industry for many years. The attitude can be summed up in a line I heard back in high school when I was learning my first assembly language on what was even then an ancient machine, the DEC-10. "You'll never need to use this because in the future all languages will be high-level like Fortran and Cobol" was the refrain of the math teachers who proctored our computer lab.

What is interesting about this quote is that it's wrong in two different ways. The first is that the languages they chose, though they are still in use today, are by far not the majority languages in software development, meaning that they weren't the be-all and end-all that these teachers thought they were. The second fallacy is the idea that what I learned in my first brush with assembly and machine code would be useless in my career, when nothing has been further from the truth. 


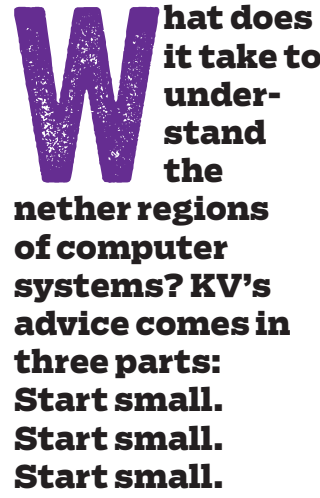

hat does understand the nether regions of computer systems? KV's advice comes in three parts: Start small. Start small. start small.
While it is true that the last piece of assembler I wrote by hand (and which wound up in a commercial product) was written 30 years ago, the lessons I learned by interacting directly with the hardware-without even the cushion of C [a.k.a. assembly with for loops]-remain with me to this day and have allowed me to track down difficult bugs, as well as significant performance problems. I've written in the past about understanding algorithms and how these expressions of problem solving relate to good performance ["Know Your Algorithms." Kode Vicious. acmqueue 16[6], 2019; https://queue.acm.orgl detail.cfm?id=3310152], but the other side of this coin is understanding how the machine on which your algorithms run actually works. What does it take to understand the nether regions of computer systems? KV's advice comes in three parts: Start small. Start small. Start small.

Start at the small end of processor and computer hardware. A modern laptop, desktop, or server system is a fantastically complex piece of equipment that for the most part grew by the accretion of features that will utterly distract anyone who is new to this area of computing. You want to start with a small system, with few features, and with a small instruction set, so that you can, hopefully, jam nearly all the details into your head at once. Symmetric multiprocessing, multilevel caches, speculative execution of instructions, long pipelines, and all the rest of the innovations in hardware that have been added to improve performance as we've hit the wall at the end of Moore's Law are important to learn-later. Very few people start learning piano by sitting down to play Mozart or Fats 
Waller, and so you should not attempt to scale the heights of a super-scaler processor on day one.

A good place to start in 2021 is with a small, cheap, embedded processor, and by this I definitely do not mean the Raspberry Pi or any of that ilk. The current Pi is based on a complex ARMv8 design that will make your head literally spin like Linda Blair's in The Exorcist. Do not start there. A better place to start is with the popular Atmel AVR chips, which are available on Arduino and other boards used by hobbyists and embedded-systems designers. These processors are eight-bit-yes, you read that correctly, eight-bit-systems much like the early microcomputers of the 1980s, and they have small memories, slow processing speeds, a small number of registers, and most importantly, a small and easy-to-remember set of assembly operations (opcodes).

These constraints actually help you learn the machine without a lot of extraneous distractions. Another advantage of this architecture is that instructions take either one or two clock cycles, depending on whether they are internal or I/O operations. Having instructions with a small, known cycle time makes it easier to think about the performance of the code you're looking at. Getting experience with performance in this way is key to being able to understand the performance of larger and more complex architectures. KV cannot emphasize enough that you want to start with an assembly language that is small and easy to understand. Go look at any data book for a big Intel, ARM, or other processor and you'll see what I mean. The AVR instruction set fits on a single page. 
Read small programs. I have given this advice to developers of languages at all levels, from high to low, but when you're trying to learn the lowest levels of the machine, it's even more important. The canonical "Hello world" program taught to all C programmers results in a binary file with millions of instructions when it's statically linked. A description of what happens when you execute it is the subject of an excellent talk by Brooks Davis at the 2016 BSDCan conference (https:I/www.bsdcan.org/2016/ schedulelevents/676.en.html].

The examples in most beginning programming tutorials for the Arduino, those that blink an LED, are the size of an example I would suggest. Later you can develop a code editor that morphs into a mail reader and web browser, which seems to be the course of all software projects over time, but for now just turn the light on and off. There are two ways to examine these small programs. The first is to look at the output of various compilers that build $C$ or higher-level code for these embedded devices, such as the Arduino IDE, or LLVM- and GNU-based crosscompilers. You can look at the code by dumping it with an objdump program, or you can look at it in a debugger if you have one that understands the AVR instruction set. The debugger is the more interesting environment because you can not only read the code but also execute it, stop it, inspect registers and memory, and the like. Both LLDB from LLVM and GDB from GNU have assembler modes, so you can even switch between a higher-level language and assembler.

Write small pieces of low-level code. That piece of assembly I wrote 30 years ago was only a couple of pages 


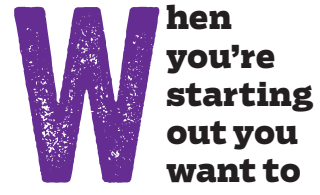

be able to hold

the entire

program in

your head if

at all possible. long, in part because what it did was simple (pulling audio data from a parallel port on a microcomputer] and because it was written using a powerful CISC (complex instruction set computer] assembly language (Motorola 68K). A CISC assembly language is a touch closer to $C$ than machine code and often has opcodes that do quite a bit on your behalf. The AVR can be considered in the family of RISC (reduced instruction set computer) processors, where each instruction is very simple, and complex operations must be built out of these. Much of the raw assembly that is still written today is along this model of a few pages of instructions.

When you're starting out you want to be able to hold the entire program in your head if at all possible. Once you're conversant with your first, simple assembly language and the machine architecture you're working with, it will be completely possible to look at a page or two of your assembly and know not only what it is supposed to do but also what the machine will do for you step by step. When you look at a high-level language, you should be able to understand what you mean it to do, but often you have no idea just how your intent will be translated into actionassembly and machine code is where the action is.

Developing these skills will take you far past blinking an LED on a hobby board. Being able to apply these same skills to larger machines, with more complex architectures, makes it possible to find all kinds of Heisenbugs [ "Kode Vicious Bugs Out." Kode Vicious. acmqueue 4[3], 2006; https://queue.acm.org/detail.cfm?id=1127862), optimize systems for power and performance, as well as understand the ramifications of low-level security attacks such as 
ROP (return-oriented programming) and buffer overflows. Without these skills, you are relegated to the cloudy upper layers of software, which is fine, until the tools or your hardware or the gods of software fail you.

Kode Vicious, known to mere mortals as George V. NevilleNeil, works on networking and operating-system code for fun and profit. He also teaches courses on various subjects related to programming. His areas of interest are code spelunking, operating systems, and rewriting your bad code [OK, maybe not that last one]. He earned his bachelor's degree in computer science at Northeastern University in Boston, Massachusetts, and is a member of ACM, the Usenix Association, and IEEE. Neville-Neil is the co-author with Marshall Kirk McKusick and Robert N. M. Watson of The Design and Implementation of the FreeBSD Operating System [second edition]. He is an avid bicyclist and traveler who currently lives in New York City. Copyright $(2021$ held by ownerlauthor. Publication rights licensed to ACM. 